\title{
The Fundamental Mechanism for the Collision/Pressure Induced Optic Effect
}

\author{
Wei-Xing Xu \\ Newtech Monitoring Inc., Oshawa, Canada \\ Email: xweixing@hotmail.com
}

How to cite this paper: $\mathrm{Xu}, \mathrm{W} . \mathrm{X}$. (2018) The Fundamental Mechanism for the Collision/Pressure Induced Optic Effect. Optics and Photonics Journal, 8, 90-97. https://doi.org/10.4236/opj.2018.84009

Received: March 15, 2018

Accepted: April 15, 2018

Published: April 18, 2018

Copyright (C) 2018 by author and Scientific Research Publishing Inc. This work is licensed under the Creative Commons Attribution International License (CC BY 4.0).

http://creativecommons.org/licenses/by/4.0/

\begin{abstract}
In this work, the fundamental mechanism regarding the collision and pressure induced optic effect is elucidated. Based on the concept of the collision-relaxation/the pressure-release induced optic effect put forth here, a new laser technology may be developed. Furthermore, our work also makes the understanding the photon involved chemical reaction become much clear and rationalized.
\end{abstract}

\section{Keywords}

Electro-Optic Effect, Magnetic-Optic Effect, Pressure-Optic Effect, Molecular Beam Technique, Laser Technology, Chemical Reaction Dynamics, Collision-Induced Optic Effect, Pressure-Induced Optic Effect

\section{Introduction}

The interaction between the light and matter has been long standing topics for scientific research. A series of important achievements in the field were made in the past, such as electro-optic effect, magnetic-optic effect and the pressure-optic effect [1] [2] [3] [4] [5]. From the electro-optic effect, the concept of the particle-wave duality of photon was finally founded and accepted by the scientific world [6], whereas the pressure-optic effect revealed the relation between the refraction/reflection of the indexes and the structure of matter [7]. The establishment of Maxwell equation indicated a milestone progress for us to understand the property of the light and founded the basis of current theory of the light [8]. And till now, the more and more applications of light have been developed, which is involved in almost every aspects in human life. For instance, the solar energy conversion [9] and electro-optic communication [10] have become the part of essential tools for the daily activity of human being. 
As part of the effort of scientific world in the field, we carefully reviewed the theory about the interaction between the light and matter. It is found that the understanding about the interaction between the light and the matter is far from perfect yet, not mention to complete. For instance, the pressure-optic effect was noticed experimentally long time ago and discussed extensively in the literatures. Based on the experimental and theoretical works, the relation between the refraction/reflection indexes and the structure of matter has been established [11]. Then the industry just takes over these scientific results and put them into application. However, to my knowledge, few of the publications give perfect answer how the defect in materials influences on the light refraction/reflection indexes of matter and what the role of the impurity in materials is taken during the interaction between the light and the matter. In most cases, we know the experiment only can give the collective results, not individual defect or impurity behaviors in the interaction between the light and the matter, but theoretically, we can explore the detail of the behaviors of the defect and impurity in the matter in the interaction between the light and the matter.

In this work, we select the simplest system, that is, a hydrogen atom to start our discussion. We hope the result in this work can help us understand some fundamental aspects regarding the collision/pressure induced optic effect.

\section{Theoretical Consideration and Discussion}

For hydrogen atom, we can start with the $\phi_{1 \mathrm{~s}}$ wave function but we put a factor, $\alpha$, onto the $\varphi_{1 \mathrm{~s}}$ wave function to mimic the behavior of the hydrogen atom experiencing the collision or under the pressure, which leads to the wave function contracting.

From the quantum mechanics, we can get

$$
\begin{aligned}
E & =\frac{\int \varphi_{1 s} H \varphi_{1 s} \mathrm{~d} \tau}{\int \varphi_{1 s}^{2} \mathrm{~d} \tau} \\
& =\frac{\int \frac{1}{\sqrt{\pi}} \mathrm{e}^{-\alpha r}\left[-\frac{1}{2}\left(\frac{\partial^{2}}{\partial r^{2}}+\frac{2}{r} \frac{\partial}{\partial r}\right)-\frac{1}{r}\right] \frac{1}{\sqrt{\pi}} \mathrm{e}^{-\alpha r} \mathrm{~d} \tau}{\int\left(\frac{1}{\sqrt{\pi}} \mathrm{e}^{-\alpha r}\right)^{2} \mathrm{~d} \tau} \\
& =\frac{1}{2} \alpha^{2}-\alpha
\end{aligned}
$$

where $E$ is the total energy of the system; $\alpha$ is the wave function contracting factor; $H$ is the Hamilton operator; $\phi_{1 \mathrm{~s}}$ is the wave function of the hydrogen atom at ground state.

From the Equation (1), we find that the energy of hydrogen atom is a function of the pressure or contracting factor, $\alpha$. The variation of $E$ with the pressure or contracting factor, $\alpha$, is shown in Figure 1.

The result in Figure 1 tells us that if the pressure applied is high enough or the wave function contracting in whatever way so great, the energy of the hydrogen atom will become very high, even higher than the energies for $\phi_{2 s}, \phi_{3 s}$, 


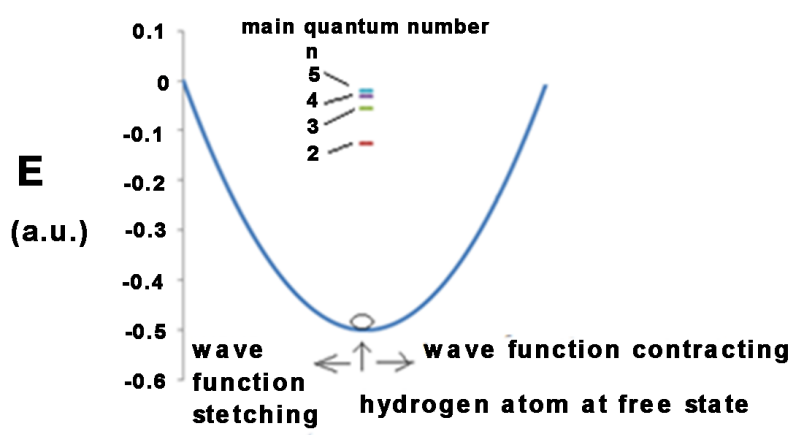

\section{elongation \\ compression
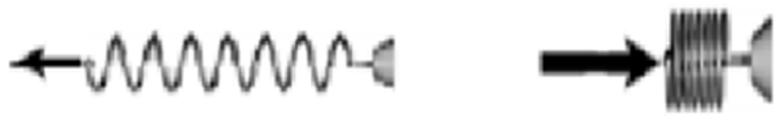 \\ equivalent classical model}

Figure 1. The illustration of wave function of hydrogen atom in contracting/stretching.

and etc. Now we concern what will happen if we suddenly release the pressure from or free the hydrogen atom. In this case, there are several channels available for this relaxation process.

Channel 1: dissipation energy through emitting infrared photon

For the convenience of our discussion, the different energy levels corresponding to the different main quantum numbers for free hydrogen atom are also marked in Figure 1. From the Figure 1, we know that if the starting point of the relaxation of the hydrogen atom located between the second lowest energy level and ground energy level, then we only can observe the infrared photon accompanying the relaxation process of the hydrogen atom.

Channel 2: dissipation energy through emitting the ultraviolet and infrared photons

If the starting point of the relaxation of the hydrogen atom located above the second lowest energy level, then, the hydrogen atom will first experience the down conversion through the vibration energy level by emitting infrared photon, then, the hydrogen atom will meet the electronic energy level, which is above the ground state energy level. Now the hydrogen atom may directly go down to the different lower energy levels (if available) by emitting the ultraviolet and infrared photons.

Channel 3: dissipation energy through the internal energy conversion

Even though we talk about the hydrogen atom here, we would like to mention the situation for multi-atoms system, such as molecule, there exists the internal energy conversion from the translational energy to vibration energy and vice versa. However, it doesn't matter in which way the internal energy conversion proceeds, finally, the system still dissipates the energy by emitting the photon (Figure 2).

From the discussion above, we find that this kind of wave function contracting, which corresponds to the collision/pressure induced optic effect, has four important aspects for us to pay attention.

\subsection{Chemical Reaction}

For the chemical reaction, we usually divide the chemical reaction into two categories: thermal reaction [12] and optical reaction [13]. When we talk about 


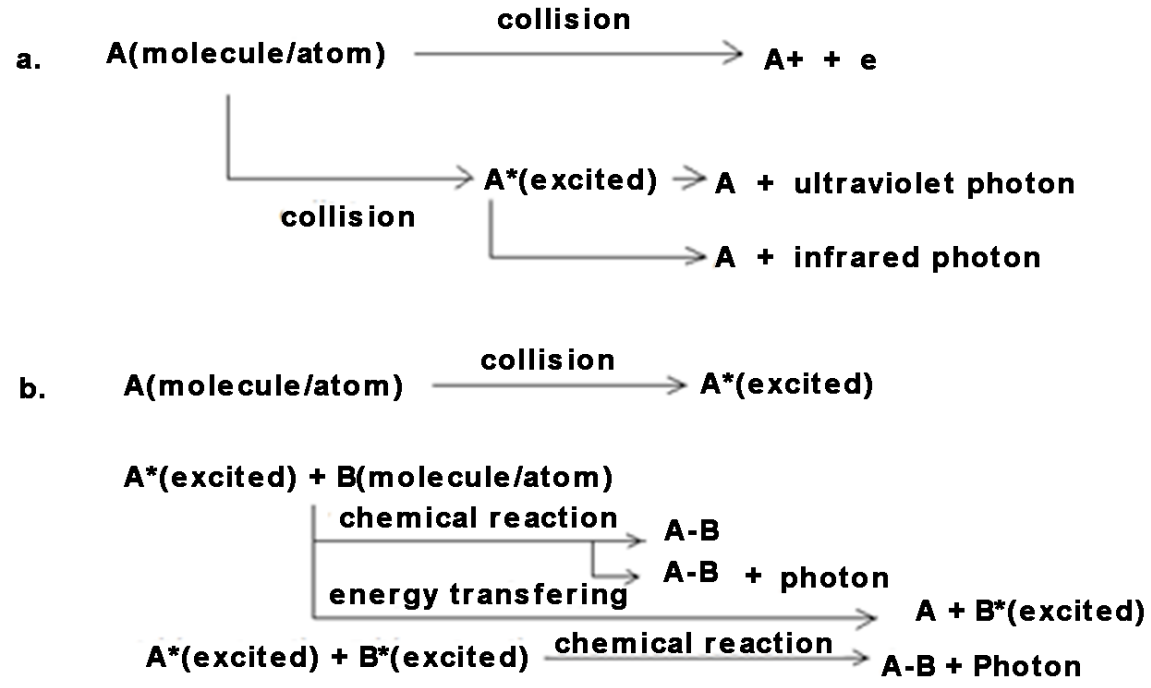

Figure 2. The illustration of the molecules/atoms in collision process.

the thermal reaction, we mean the reaction proceeds with giving off or absorbing the heat. In order to keep the reaction continuing, we have to heat or cool the system to keep the system staying at the temperature required by the proceeding of the chemical reaction. For optical chemical reaction, usually we mean that we have to first excite the molecule or atom to the excited state by absorption of photon, then the chemical reaction occurs.

All these kinds of chemical reaction have been understood quite well, at least theoretically. Following we would like to consider the chemical reaction which is different from the chemical reaction cited above.

Based on our discussion regarding the behavior of the hydrogen atom under the orbital contracting, we know that we can promote the molecule or atom to high energy level by applying the pressure onto the system, then releasing the pressure from the system, the energy of the system will redistribute among the molecules or atoms. One important process for us to stress is the molecule or atom will dissipate the energy by emitting photon. This emitted photon may just go away from the system or excite another molecule or atom to the excited state. This kind of energy redistribution by emitting and absorbing photon among the reactants can make the chemical reaction occur. The special point for this kind of chemical reaction is in that it doesn't need heat/cool the system to the temperature required by the chemical reaction nor to promote the molecule or atom to the excited state by the light radiation. Instead the molecule or atom is promoted to the high energy level just by applying the pressure onto the system. Sometime the photon can be detected as by-product, not the reaction starter as usual in optical-chemical reaction. Sometime no photon can be detected if the photon totally be consumed to promote the molecule or atom to high energy state. In this case, the energy transferring between the molecules or atoms become more efficient, no extra energy is lost by emitting extra photon. But the energy redistribution among the molecules and atoms is still realized by the 
photon emitting and absorbing, even though no photon be detected.

From the discussion above, we can define this kind of chemical reaction as orbital contracting-induced optical reaction. Due to the increasing pressure of system is the easiest way to cause the orbital contracting, therefore, we also can call this kind of reaction as the pressure-induced optical reaction.

\subsection{The Chemical Reaction Dynamics Study}

From the literatures, a lot of researches about the chemical reaction dynamics are performed based on the molecular beam technique [14]. Conventionally, the molecular beam technique is to study the molecular chemical dynamics by the collision of reactants and the distribution of the products. Based on the molecular beam technique, a lot of chemical reaction mechanisms have been elucidated. However, to my knowledge, one important case has been missing, that is, during the process of collision of two or more molecules/atoms, the chemical reaction may involve in the photon emission/absorption. Simply speaking, the conventional molecular beam technique only discusses the internal energy conversion from the translational energy to the vibration energy and vice versa. Whereas we discussed here is the photon emission/absorption induced by the collision or pressure. In fact, the collision of molecule/atom and applying the pressure onto the system involved in the same fundamental mechanism, that is, the wave function of molecule/atom contracting. For the photon emission induced by molecule/atom collision, it is the translational energy of molecule/atom changes partly to the photon energy, partly to the rotational/vibration energy. We search the literatures, there is no publications regarding the photon emission by the molecule/atom collision reported, whereas for the photon emission induced by the pressure, most of publications only talk about the pressure induced the change of light refraction/reflection indexes, instead of the collision/pressure induced photon emission.

In reality, the molecular collision induced photon emission and the pressure induced photon emission are very popular phenomenon around us. For example, if the energy of two hydrogen atoms in collision is high enough, the electron in hydrogen atom may totally be kicked out of the hydrogen atom (Figure 2); if the energy of two hydrogen atoms in collision is not high enough, the electron in hydrogen atom will be kicked from the lower energy level to higher energy level, then, the electron will relax back from the higher energy level to lower energy level, the photon will be emitted. For the pressure induced photon emission, the experiment becomes so easy that even a middle school student can demonstrate it. For example, to hit a piece of match as hard as possible with hammer, then we will observe the photon emission by the naked eye. These phenomena of the collision/pressure induced photon emissions are much easy to observe, therefore, there is no reason for scientific world to ignore it.

\subsection{The Expectation Value of $<r>$}

From the discussion above, the wave function contracting due to the colli- 
sion/pressure will make the total energy of system increase, then, following the system relaxation, the photon will be emitted. We use the contracting factor, $\alpha$, to describe the wave function in contracting. From the quantum mechanics, we know the expectation distance of the electron from the nucleus can be described by $\langle r\rangle$, which is calculable if the wave function is available (Equation (2)). Therefore, $\langle r\rangle$ can be used to measure the distance between the atom/molecule in the collision or under the pressure. The $\langle r\rangle \sim \alpha$ relation for hydrogen atom is shown in Figure 3 and each energy level corresponding $\langle r\rangle$ also marked in Figure 3.

$$
\begin{aligned}
<r>= & \frac{\int \varphi_{1 s} \cdot r \cdot \varphi_{1 s} \mathrm{~d} \tau}{\int \varphi_{1 s}^{2} \mathrm{~d} \tau} \\
& =\frac{3}{2 \alpha}
\end{aligned}
$$

where $\alpha$ is the wave function contracting factor; $\phi_{1 \mathrm{~s}}$ is the wave function of the hydrogen atom at ground state; $\langle r\rangle$ is the expectation distance between the electron and nucleus in hydrogen atom.

For example, if we detect the photon corresponding to the transition between the certain energy levels of hydrogen atom, then, we know the closest distance between two hydrogen atoms should be $\leq 2\langle r\rangle$. Only meeting with this condition, the system can emit the photon corresponding to the transition between the certain energy levels of hydrogen atom. This work here offers a way to mimic the chemical reaction by collision or under pressure and make the energy utilization become more efficient.

\subsection{The Possibility for Developing New Laser Technique}

Here we would like to explore a possibility to develop new laser technology based on the discussion above. In order to make a system lasing, we first have to pump the electron to high energy level and realize the energy level inversely

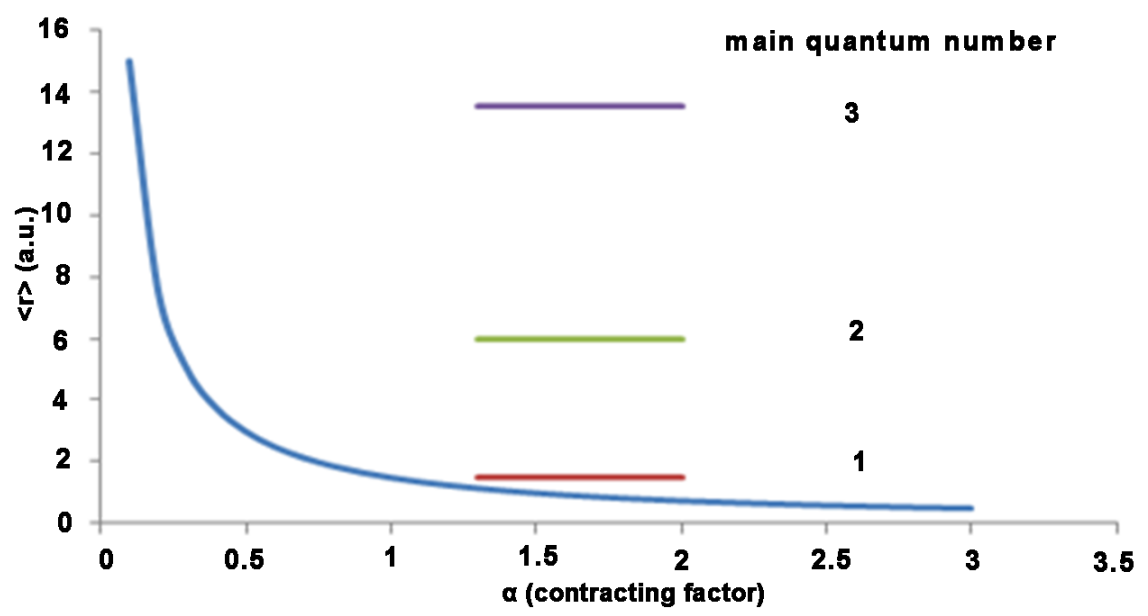

Figure 3. The illustration of the dependence of the $\langle r\rangle$ on the wave function contracting factor. 
popularized by the electron. From the discussion above, the electron inversely popularization can be realized by the collision-relaxation or the pressure-release cycle. Even though at moment, the realization of the electron inversely popularization by the collision-relaxation or the pressure-release cycle is just at the concept stage, at least there is no theoretical difficulty for us to realize the electron inversely popularization by the collision-relaxation or the pressure-release cycle. Most important is that the wave length of the laser in this case is tunable, we just need to set the system working in different collision-relaxation or the pressure-release cycle range as we discussed above. Therefore, we can say our work here opens a bright avenue to develop a new laser technology by the collision-relaxation or the pressure-release cycle.

\section{Conclusion}

In this work, we take the hydrogen atom as an example to study the wave function contracting and found that the wave function contracting will lead to increase of the total energy of system. If we allow the system to freely relax, under certain condition, the system will emit photon. This kind of wave function contracting can be realized in a simple way, such as the collision among the molecules/atoms or applying the pressure onto the system. The collision-relaxation or the pressure-release induced the photon emission represents an important but different process from the conventional molecular beam technology, which is unreasonably ignored before. This kind of the collision-relaxation or the pressure-release induced the photon emission also offer a new way for us to draw some chemical reaction information, such as the closest distance between the molecules/atoms in reaction. Furthermore, our work here points out a possibility to develop new laser technology by the collision-relaxation or the pressure-release cycle, which will find a wide application in scientific research and industry. Further work in this direction will be presented later.

\section{References}

[1] Castles, F. (2015) Linear Electro-Optic Effect Due to High-Order Spatial Dispersion. Physical Review A, 92, Article ID: 063804. https://doi.org/10.1103/PhysRevA.92.063804

[2] Consoli, F., De Angelis, R., Duvilaret, L., Andreoli, P.L., Cipriani, M., Cristofari, G., Di Giorgio, G., Ingenito, F. and Verona, C. (2016) Time-Resolved Absolute Measurements by Electro-Optic Effect of Giant Electromagnetic Pulses Due to Laser-Plasma Interaction in Nanosecond Regime. Scientific Reports, 6, 1-14.

[3] Robinson, T.S., Consoli, F., Giltrap, S., Eardley, S.J., Hicks, G.S., Ditter, E.J., Ettlinger, O., Stuart, N.H. Notley, M., De Angelis, R., Najmudin, Z. and Smith, R.A. (2017) Low-Noise Time-Resolved Optical Sensing of Electromagnetic Pulses from Petawatt Laser-Matter Interaction. Scientific Reports, 7, Article Number: 983. https://doi.org/10.1038/s41598-017-01063-1

[4] Bader, S.D. and Erskine, J.L. (1994) Magnetic-Optical Effects in Ultrathin Magnetic Structures. In: Heinrich, B. and Bland, J.A.C., Eds., Ultrathin Magnetic Structures II, Springer, Berlin, Heidelberg. 
[5] Gorachand, G. (1998) Model for the Pressure-Optic Coefficients in Optical Materials. Physical Review B, 57, 8178. https://doi.org/10.1103/PhysRevB.57.8178

[6] Einstein, A. (1905) On a Heuristic Viewpoint Concerning the Production and Transformation of Light. Annalen der Physik, 17, 132-148. https://doi.org/10.1002/andp.19053220607

[7] Waxler, R.M. and Weir, C.E. (1965) Effect of Hydrostatic Pressure on the Refractive Indices of Some Solids. Journal of research of the National Bureau of Standards, Section A: Physics and Chemistry, 69A, 325-333. https://doi.org/10.6028/jres.069A.033

[8] Maxwell, J.C. (1865) A Dynamical Theory of the Electromagnetic Field. Philosophical Transaction of Royal Society of London, 155, 459-512.

https://doi.org/10.1098/rstl.1865.0008

[9] Tabah, B., Pulidindi, I.N., Chitturi, V.R., Mohana, L., Arava, R. and Gedanken, A. (2016) Utilization of Solar Energy for Continuous Bioethanol Production for Energy Applications. RSC Advances, 219, 24203-24209. https://doi.org/10.1039/C6RA00389C

[10] Shi, Y.Q., Zhang, C., Zhang, H., Bechtel, J.H., Dalton, L.R., Robinson, B.H. and Steier, W.H. (2000) Low (Sub-1-Volt) Halfwave Voltage Polymeric Electro-Optic Modulators Achieved by Controlling Chromophore Shape. Science, 288, 119-122. https://doi.org/10.1126/science.288.5463.119

[11] Waxler, R.M. and Weir, C.E. (1963) Effect of Pressure and Temperature on Bureau of Standards. Journal of Physical Chemistry A, 67A, 163-171.

[12] Yin, X., Wu, J.B., Li, P.P., Shi, M. and Yang, H. (2016) Self-Heating Approach to the Fast Production of Uniform Metal Nanostructures. ChemNanoMat, 2, 37-41. https://doi.org/10.1002/cnma.201500123

[13] Bassham, J.A. (1957) The Path of Carbon in Photosynthesis. Prentice-Hall, Bailey \& Swinfen.

[14] Estermann, I. (1946) Molecular Beam Technique. Reviews of Modern Physics, 18, 300. https://doi.org/10.1103/RevModPhys.18.300 